\title{
Structuring a virtual environment for sport training: A case study on rowing technique
}

\author{
Emanuele Ruffaldi*, Alessandro Filippeschi \\ Scuola Superiore S. Anna, Pisa, Italy
}

\section{A R T I C L E I N F O}

\section{Article history:}

Available online $\mathrm{xxxx}$

\section{Keywords:}

Training

Skill

Motion analysis

Vibrotactile

Feedback

\begin{abstract}
A B S T R A C T
The advancements in technology and the possibility of their integration in the domain of virtual environments allow access to new application domains previously limited to highly expensive setups. This is specifically the case of sport training that can take advantage of the improved quality of measurement systems and computing techniques. Given this the challenge that emerges is related to the way training is performed and how it is possible to evaluate the transfer from the virtual setup to the real case. In this work we discuss the aspect of system architecture for a VE in sport training, taking as a case study a rowing training system. The paper will address in particular the challenges of training technique in rowing.
\end{abstract}

(C) 2012 Elsevier B.V. All rights reserved.

\section{Introduction}

Training in Virtual Environments has received much attention in the past due to the possibility of tracking subject performance, recreating working conditions, and introducing effects not easily reproducible in real training conditions [1]. At the same time the adoption of a Virtual Environment (VE) raises the issue of the transfer of performance from the task performed in the VE and the real one. A specific challenge arises when the task to be trained has a sensorimotor component that is prevalent over the cognitive one. One domain of VE training that is receiving attention is the one of sport [2], because it challenges technology and research: the former in the aspect of fast pacing action and high forces, the latter in the transfer of training.

This work addresses these two challenges and aims at presenting a methodology and architecture for tackling training in VE with a specific case study in the domain of sport. The proposed methodology first focuses on the understanding of the trained task. Then it concentrates on technological issues. It highlights in particular the importance of a digital representation of the training skill that is fundamental for supporting the system logic during training.

The case study of this work will be the SPRINT rowing demonstrator [3] developed inside the SKILLS European project. This demonstrator addresses several aspects of rowing and it shows how the proposed methodology and architecture allows covering the different skills involved in such a complex activity. In particular the paper will present first the design requirements

\footnotetext{
* Corresponding author.

E-mail address: e.ruffaldi@sssup.it (E. Ruffaldi).
}

for the definition of such a system, then the proposed architecture and cases of evaluation performed on the platform.

\section{Design criteria for a multimodal training platform}

The creation of a VE platform for training involves, as an initial step, a study of the task to be trained for understanding which aspects are both interesting and technologically feasible and which aspects will be the focus of the system itself [4]. Having understood the focus and the characteristics of the task the user experience is designed, expressed in terms of the interfaces and simulation elements that the user will face. The next phases are more inherently technical and allow us to precisely design the training system. In particular four phases can be identified, and they will be the focus of the discussion of this paper:

1. The design of the training platform.

2. The development of a digital representation of the features and the skills involved in the tasks the system aims at training.

3. The design of the information exchange with the user and the setup of the training protocols tuned on a user's abilities and goals.

These elements constitute with the trainee the training loop in which information flows in order to improve a user's performance.

The platform embeds the interfaces with the human, therefore it provides the hardware for the user-system information exchange, thus physically closing the training loop. The training platform has to include all the features that the task analysis showed to be worth for training. At the same time features that cannot be included with a sufficient goodness to be positively effective for training have to be discarded, as they would indeed be detrimental for training [5]. Typical components of such platforms are haptic devices, tools 


\section{$A R T C$ CLE IN PRESS}

for capturing a user's performance (e.g. motion tracking systems and force sensors), visualization systems and audio devices. At this stage the information flow is both from the system to the user (force rendering, visualization, etc.) and from the user to the system (motion capturing, force capturing, etc.). The simulation of the task is also a fundamental part of the training loop placed in the middle of the information exchange. On the one side it allows us to tune force rendering. On the other side it provides information for evaluating a user's performance.

The digital representation of the skills is the core of the training system. It synthesizes the key aspects of performance in a quantitative way thus allowing a quantitative and objective evaluation. The digital representation is the result of experts' performance analysis and presents some challenging aspects. The first is the sought-after meaningful indices that are actually affected only by variables that influence performance quality, being insensitive to others. The second is the scalability: indeed indices should not be affected by a user's characteristics like size or weight. The information is organized at high level by representing the semantics of training decomposed in the different timing cycles, while at low level information is characterized by the sensor and simulation data augmented with performance indices. Flexibility, performance and reuse are three key elements of such a representation. The first deals with the adaptation of different algorithms and solutions for characterizing the subject. Performance deals with the possibility of efficiently computing indices, to being available immediately after performance, thus allowing users to be provided with feedback as soon as they do the task. Finally reuse deals with the possibility of creating libraries of analysis elements that can be reused across different training protocols and across tasks.

The information exchange has to be carefully planned both from the user to the system and from the system to the user. The userto-system exchange is provided by the sensors implemented and it determines the information about a user's performance that the system is aware of. The system-to-user exchange allows us to provide users with feedback and stimuli that are considered useful for training. From this point of view the system-to-user exchange design has to take into consideration the different sensory channels available for the user and the instruments the platform is provided with to give feedback. At this stage all the advantages of multimodal platforms should be exploited to the most to speed up training and consolidate learning. In particular, since humans rely mostly on sight, visual display devices are of fundamental importance in VEs and their potential and effectiveness for training (also in sport) has been shown. A discussion about the design of feedback for sport training can be found in previous work [6].

The training protocols manage the times and the timing of workout and rest time, at the same time tuning the frequency and the amount of feedback. The training protocols are tuned according to the task, the goal and the user's initial skills. In sport, in addition to common motor and cognitive skills, physiological status has to be considered, in fact, many sports require users to improve their physiological status before carrying out highly demanding tasks. Therefore, all these three variables have to be considered at a time to avoid training not to be effective or, worse, to cause injuries to the user. A large part of the research in the work presented here focuses on the development of what were called accelerators, that are combinations of information exchange elements and protocols aimed at shortening the training process and at stabilizing learning. The evaluated accelerators provide training units that may last from a few days to several weeks. These units do not aim at substituting existing training, but they are developed to be integrated in the current training protocols.

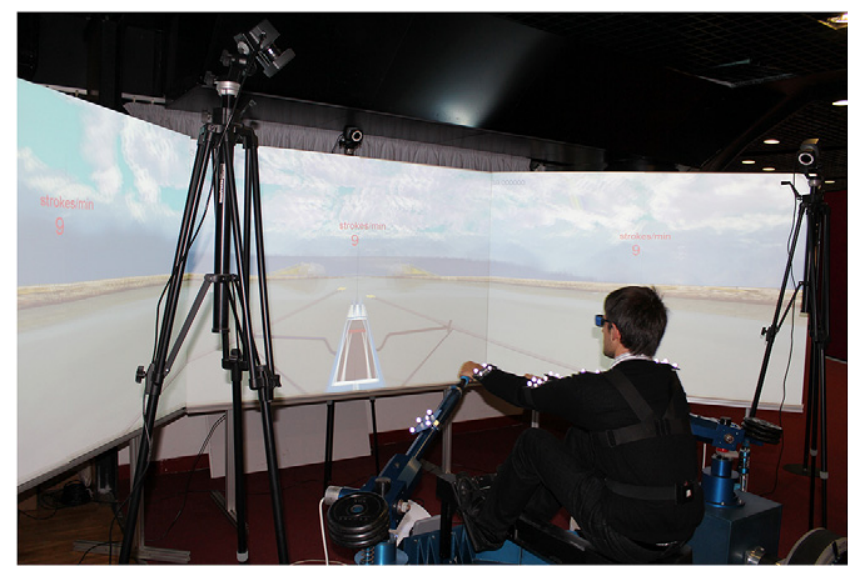

Fig. 1. The SPRINT rowing training system presented in an immersive configuration with three stereographic screens and motion capture.

\section{The SPRINT rowing training system}

In this section the rowing training system called SPRINT is shown as an example of multimodal platforms designed with the previously mentioned principles. The design of each part is the result of the trade-off between training/simulation requirements and resources (in terms of both computational and system's complexity) expenditure. The system indeed aims at training, at the same time being affordable for potential trainees.

The ultimate goal of rowing is to win races. It requires crews to cover the $2000 \mathrm{~m}$ racing distance as fast as they can. The task analysis moved from this point and finally showed that rowers have to improve many capacities and skills. The most important ones are: high muscular power, high aerobic and anaerobic capabilities, ability to manage gesture in order to achieve high biomechanical efficiency, ability to manage own energy even when he/she acts expressing high powers and stroke rates, and ability to coordinate with teammates. Since only a few of these skills could be quantitatively evaluated, current training protocols aim mostly at improving physiological status. SPRINT was hence developed to also train the other skills. In particular, three main training areas were selected and studied: technique, energy management and team coordination. Platforms, digital representation, information exchange and protocols were developed to train each of these three areas.

\subsection{Interfaces}

SPRINT allows combining several devices to obtain different platforms. In particular two mechanical platforms and two visualization systems are available. The full mechanical platform is provided with two oars and allows differently sized users to train both sculling and sweep rowing. The lightweight mechanical platform is a Concept2 ergometer (Concept2, Morrisville, VT) that allows users to row with a limited range of kinematic features yet providing them with a good force rendering. The most common visualization system is a LCD screen placed in front of the user, but the system can be reconfigured to be placed in a CAVE [7] like a 3D Virtual Environment. Fig. 1 shows the full platform in an immersive setup, whereas 2 shows the lightweight platform. Mechanical platforms are affected by several limitations since, according to [5], only features that were considered worthwhile for training, or that could be correctly implemented without excessively complicating the system, were kept. For example, both lightweight and full platforms can be used for energy management training, whereas only the full platform is suitable for technique optimization training. Another example is provided by the rotations of the boat (i.e. pitch, roll and yaw), that were neglected because 


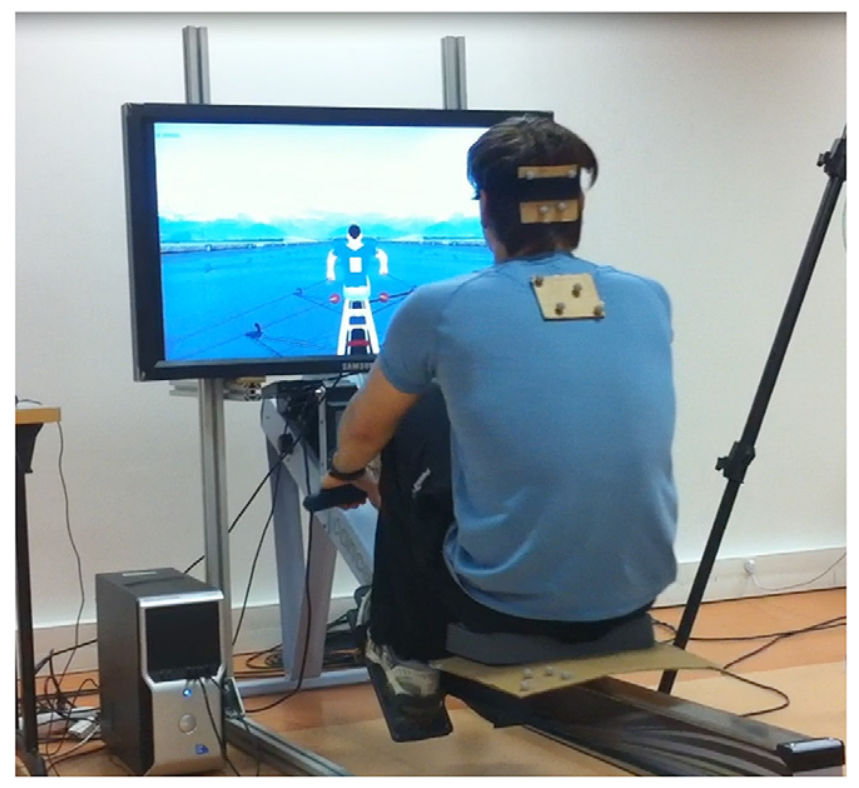

Fig. 2. The SPRINT lightweight platform.

their implementation would make the system too complex if we want to simulate them correctly. External devices may be added to both platforms for capturing purposes: the VICON motion tracking system allows us to accurately estimate a rower's motion; Cosmed $\mathrm{k} 4$ records oxygen consumption and other physiological measurements; infrared sensors can be integrated for a light version of motion tracking.

\subsection{Task modeling}

Modeling the rowing task has two main purposes. The first and most important is to provide information about the outcome of the user's performance thus enhancing the system's evaluation capabilities. The second is to simulate the task in order to develop a real-task-like environment, whose increased plausibility enhances the system's effectiveness in conveying information to the user. The rowing task's main components are the rowers, the boat (and the oars), the water and the air. Each of them can be modeled at different levels of detail, hence the criteria explained in Section 2 were applied. Three models of the rower were developed to satisfy training purposes and according to the system setup. The most complex is a full body model that provides forces and displacements of each articulation, it is used for offline analysis of a user's performance and only if the full body motion tracking is available. The second is a less detailed one. Its main advantage is that it can be run online, thus allowing a real-time evaluation of performance. It requires the shoulder's motion tracking in addition to seat and handle positions. The third is the simplest and requires only seat position. The boat was modeled according to the decisions taken for the interfaces: yaw and roll were neglected, whereas pitch and heave, that do not complicate the simulation too much, but allow us to have indices of motion effectiveness and efficiency, were kept. Modeling of fluid-structure interaction as well as modeling of the wind and the waves were neglected as they were not considered worthwhile for training enough to justify the resources demanded. For the same reason both hull and oars were considered rigid bodies.

\subsection{Digital representation}

The systems provide scores and indices related to the three training areas spotted in the task analysis. Most of these indices are available immediately after the performance to allow online feedback, that was shown to be very effective for training motor skills [8].

The first step of the analysis is the segmentation: according to the current training protocols the largest cycles last four years (the time between two Olympics). Then there are smaller cycles of months, weeks and days that end in the blocks of a single workout during a given day (e.g. to carry out a five minute workout of nonstop rowing on Monday). At a more detailed level there is stroke segmentation: since rowing is a periodic task whose unit cycle is the stroke, captured and processed data are segmented by stroke. A more detailed segmentation is carried out within the stroke: four or more phases were spotted to break down performance into units useful for the evaluation.

Given this structure for the performance breakdown, several indices were developed for the evaluation for each training area.

Technique Technique evaluation is the most complex and comprises several indices related to motion timing, spatial accuracy and intrapersonal coordination. Timing indices are the least affected by users' variability. However, they are the most affected by capturing errors, since they require an accurate knowledge of when captured actions were performed. At stroke level users are provided with the stroke pace (number of strokes per minute), while at phase level the ratio between pull and recovery phases is calculated. When motion tracking is available, legs, back and arms motion onset timing indices based on expert performance analysis are calculated. Spatial indices are the most influenced by user size but the least by timing of capturing. A user's size dependence was avoided by developing calibration procedures, as for oar handle trajectory accuracy, or by selecting variables least affected by size, such as back angle or the arm-forearm plane orientation during the arm pull [9]. Body limbs motion onsets timing is an example of an index related to intrapersonal coordination. Timing, and spatial accuracy as well as intrapersonal coordination underlie the performance of technique faults. Machine learning techniques were applied to experts' performance when asked to voluntarily perform faults. These indices allow us to spot main faults of rowing technique almost independently of the user size [10]. Most of these indices (all but stroke pace and pull-recovery ratio) require an accurate simulation and capturing of the rower motion, therefore they require the full platform and a motion tracking system to be employed.

Energy Energy management is evaluated at workout level. Boat speed profile as well as power output and, when available, oxygen consumption, are checked to match the expert's profile in order to provide indices of energy management efficiency. One index taken into account is the distance to a virtual opponent moving at a target speed calculated based on a user's level [11]. These indices do not require accurate modeling of rower motion and can be calculated also in the lightweight platform without a motion tracking index. External devices for physiological variables capturing increase the indices available for this area of training; they are strongly user dependent, therefore these devices require an initial calibration.

Team Team coordination is evaluated by comparing a user's motion to a virtual mate reference one, which can be artificially generated by the trainer or based on a real expert performance. Speeds and positions are suitably combined to have an index of coordination. Team 


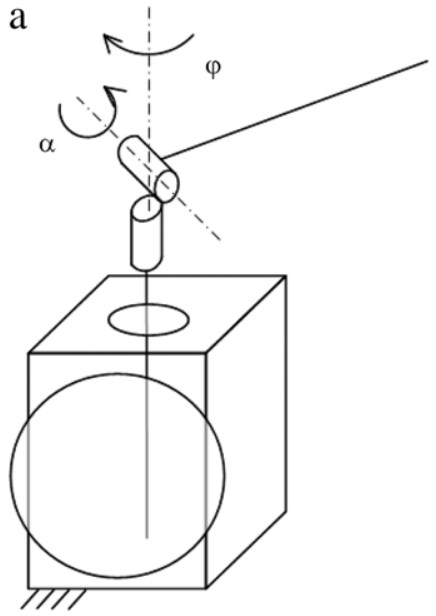

b

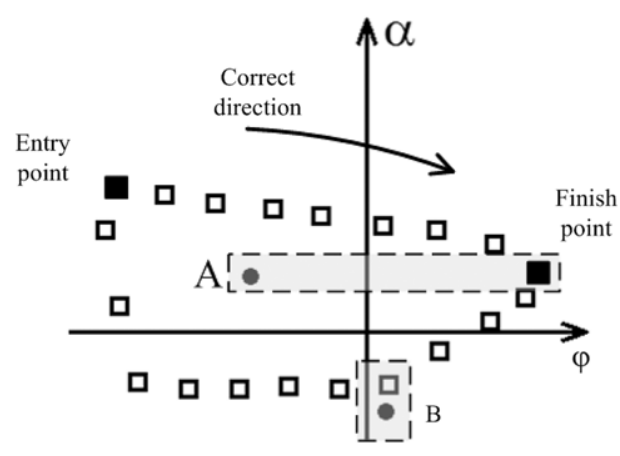

Fig. 3. (a) The $\varphi$ and $\alpha$ angles. (b) Example of distance selection for scoring in case of wrong (A) and correct (B) direction.

coordination can be evaluated both on the lightweight and the full platforms, the latter providing more variables for calculating coordination indices. In case the user is required to coordinate with an artificially generated motion pattern, or if the real rower size differs from the user's ones more than the platform regulations allow for compensation, the system is initially calibrated.

\subsection{Training}

Training on the SPRINT platform was designed following a temporal structure: exercises comprise test, workout and rest time blocks. These elements were combined depending on trainee's expertise, physical status and on training task requirements. Variables for the training design were multimodal feedbacks and load tuning. SPRINT allows us to give users feedback with visual, vibrotactile, haptic and audio modalities. Visual feedback are indicators about trajectory accuracy, gauges about power output, indicators of the distance to a virtual opponent, indicators of the coordination with a virtual mate as well as numerical indicators of the stroke pace or of the boat speed. Symbolic indicators (such as gauges, numbers or playback of performance in graphs) or indicators embedded in the scene (like arrows or even embodied by VE agents as in the case of the virtual mate coordination) were selected according to their supposed and evaluated effectiveness for training. Vibrotactlie feedback was largely used for refining performance in technique training. Bracelets worn on the wrists and on the back provide feedback about handle trajectory accuracy, body limbs motion onsets timing correctness as well as about technique faults. Load tuning is a simplification of the task, that is necessary, for example, to avoid fatigue in technique training for novices.

Two days to six weeks protocols in which users are provided with online and offline feedback are currently available in SPRINT. Longer protocols are for intermediate rowers and they are suitable to be integrated with currently existing training protocols. Shorter protocols are mostly for novices. Adaptation of feedback is adopted in both cases to reduce a trainee's dependency on feedback. In the following section examples of accelerators for technique and energy management training are presented.

\section{SPRINT evaluation}

In this section training of oars' trajectories mastery and body motion timing are shown as examples of the training accelerators available on SPRINT and of the training scenarios that can be implemented. The decision taken from the platform selection to the protocols definition are explained highlighting the application of the design criteria.

\subsection{Trajectory training}

The task analysis showed that the rowing gesture requires the coordination of several body limbs with, however, only one simple goal: make the oars cover the right cyclic trajectory in the right direction avoiding stops. This goal is easily accomplished by already experienced rowers, but it is not trivial for beginners. Therefore the first step of rowing technique training is dedicated to novices and aims at making them move the oars correctly, that is to move hands correctly taking into account the oar's mechanical constraints.

Training of the gesture requires all kinematic features, therefore the full platform is selected. Since only oars trajectories are worthwhile for training motion tracking is not necessary. At this stage modeling of the outcome of a trainee's performance is not needed, as only the trajectory is considered. Therefore we only make use of the simplest boat and rower's models, aiming only at correct visualization.

Expert trajectories were captured and used as the reference for novices, thus allowing for calculating indices for evaluation. Oars positions and orientations depend mostly on two angles, namely $\alpha$ and $\varphi$. The first one determines handle vertical displacement, the latter the horizontal one. Euclidean distances of the performed trajectory from the pattern in $(\varphi, \alpha)$ were used to score performance, however, the score took into account also the direction the cycle was covered and stops. A state machine based on oars orientation allowed us to have a 20 phase segmentation of the stroke that was used for detecting direction and stops. In case of correct direction performance score decreased as the distance from the pattern decreased, otherwise it was determined by the maximum distance of the current sample to the reference's entry and finish samples (see Fig. 3). The main issue to have a fair evaluation is the dependence on a user's size. It was solved by calibrating the system: the $\alpha$ angle is determined by system geometry, so it is calculated given the regulations of the mechanical platform. The $\varphi$ angle requires users to carry out a calibration procedure. They were asked to go at the beginning and at the end of the pull phase, where $\varphi$ is respectively at its minimum and maximum values. Given the ranges of $\alpha$ and $\varphi$ a user's performed trajectories were linearly scaled to be comparable 

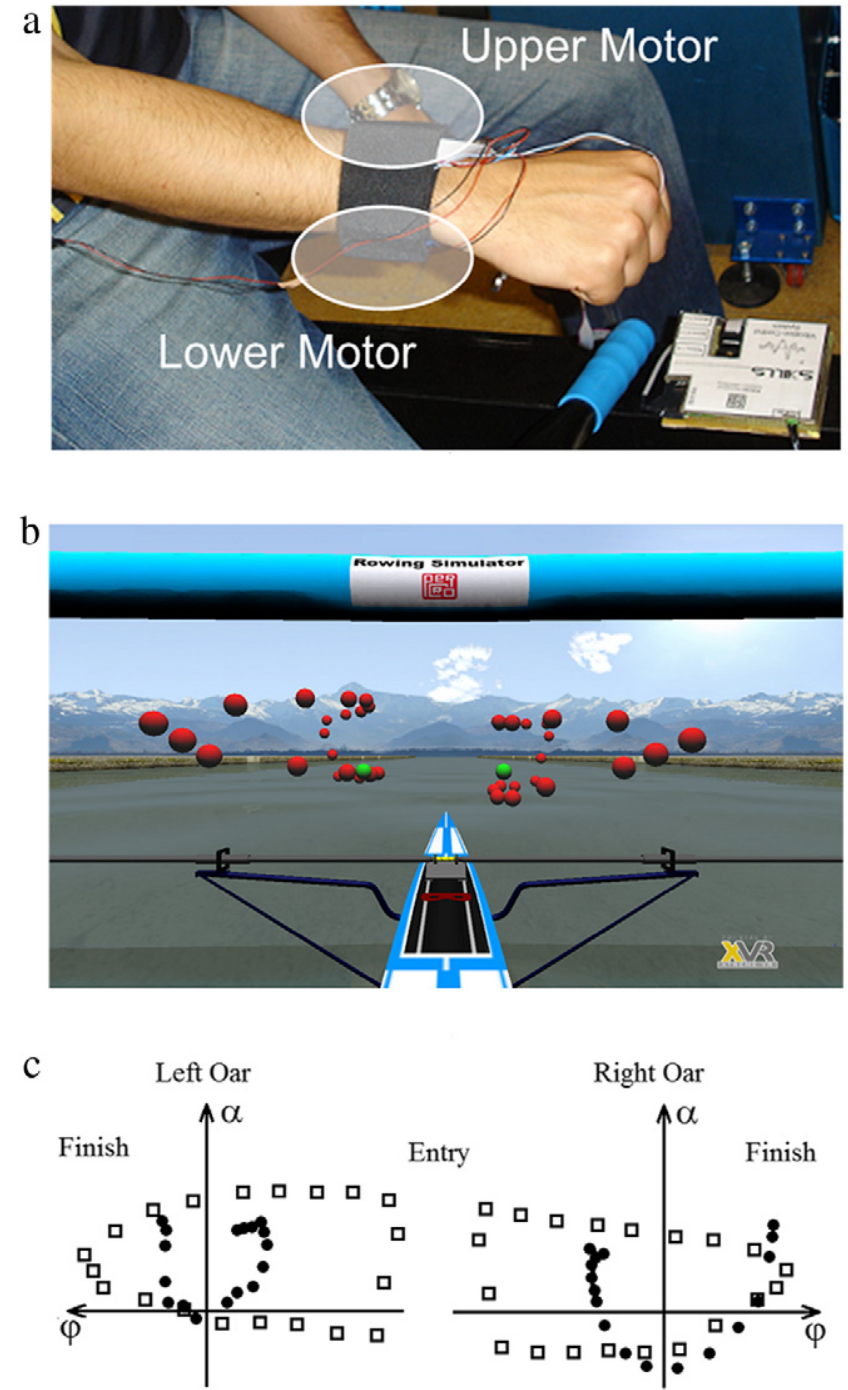

Fig. 4. (a) Vibrotactile feedback device. (b) Online visual cue immersed in the VE. (c) Offline summary of performance for the trajectory training. Empty squares represent correct points to be followed, whereas filled circles are samples of the actually performed trajectory.

with the reference one. The performance index so calculated is immediately available to provide online feedback.

In this scenario training blocks are one minute long during which users row with full load but at low pace to avoid fatigue which is an important issue for novices. A visual cue is always available to have a comparison of ongoing performance against the correct one. Visual cues are shown in an LCD screen as elements of the Virtual Environment, where reference and current hands trajectories were superimposed onto the virtual boat and oars. At the same time, online vibrotactile feedback is provided on the wrists by means of bracelets containing vibrating motors, one on the top of the wrist and the other on the bottom. The upper motor starts vibrating when the hand is over the ideal trajectory, and vice versa for the lower motor. The intensity of vibration is an exponential function of the error. It is crucial that the training loop (capturing-analysis-rendering) be quick in order to avoid feedback to be misleading or to be ignored by the user [12]. A summary of the block performance is available after each training block as a plot showing performed against correct trajectory for each oar. Visual cues and the vibrotactile feedback device are shown in Fig. 4. Feedback frequency as well as its intensity were chosen according to the literature about motor skills learning (e.g. [13]). The objective is to have an effective feedback avoiding, at the same time, trainees to become dependent on it. However, it is possible for the coach and the trainee to set them. Protocol length was decided to avoid novices getting tired: fatigue is indeed likely to cause trainees to make errors and to stabilize on an incorrect gesture, thus being detrimental for training.

In the following the experiment carried out to evaluate effectiveness of this training accelerator is summarized. More details are available in [14]. The experiment was performed by a group of 25 healthy males (aged 20-26) who had no previous rowing experience. Participants were asked to perform the whole rowing cycle (stroke) and were evaluated as previously explained. The provided feedback was evaluated by means of pre-post assessment test blocks, carried out without feedback. The participants' goal was to row as close as they could to the reference model. In this experiment speeds were not constrained neither evaluated. However, participants were asked to row faster than 8 strokes per minute. Three different online training conditions were tested: only vibrotactile feedback (VIB), only visual cue (VIS), and vibrotactile feedback and visual cue (VIB + VIS). A fourth group with no feedback (NF) was tested as the control group. In addition, during rest time, all participants were provided with a static summary of their last block performance. The experiment was carried out on the rowing demonstrator. The Virtual Environment shown in Fig. 4 was displayed during workout trials. Red and green balls were shown only for VIS and VIB + VIS groups' participants. All wore on their wrists two belts equipped with vibrating motors (see Fig. 4), regardless of the training condition. The experiment was carried out during two consecutive days. On the first day each participant performed a preliminary test for assessing their initial level, then they carried out ten training blocks, and finally, an assessment test block was performed. During the second day six training blocks and a final test block were carried out. Both tests and training blocks lasted one minute. The participants were given one minute of rest time between blocks. Online feedback was provided only on the first day and only during the training trials; tests were performed without feedback. During rest time the summary of the last block performance was presented every second block. The previously mentioned error was computed for each point of the trajectory. Phase, stroke, and block errors were respectively the average of errors of that phase, stroke or block. The first index we used to compare different training conditions was the mean block error applied across participants who experienced the same training condition. In particular the decrease of such an error, that is the improvement in performance, between two blocks was considered. Fig. 5 shows improvements between post training and initial tests for the four groups. Results lead to the conclusion that trajectory training is effective and that the combination of vibrotactile feedback and visual cues is the best one for enhancing learning and for the retention of trajectory mastery.

\subsection{Timing training}

The most important phase of the rowing cycle is the drive (when rowers propel the boat), that is characterized by leg drive, back swing and arms pull, to be performed in this order at the correct time to maximize drive phase effectiveness. Literature and recent analyses (see $[15,9]$ ) allow us to say that the temporal structure of body limbs motion onset during the drive phase is one important feature for determining technique effectiveness. Timing training is more suitable for intermediate rowers rather than for novices as it aims at refining an already acquired motion pattern. Therefore, the design of body limbs motion training for novices is a challenging task that requires us to exploit at most the system capabilities. 

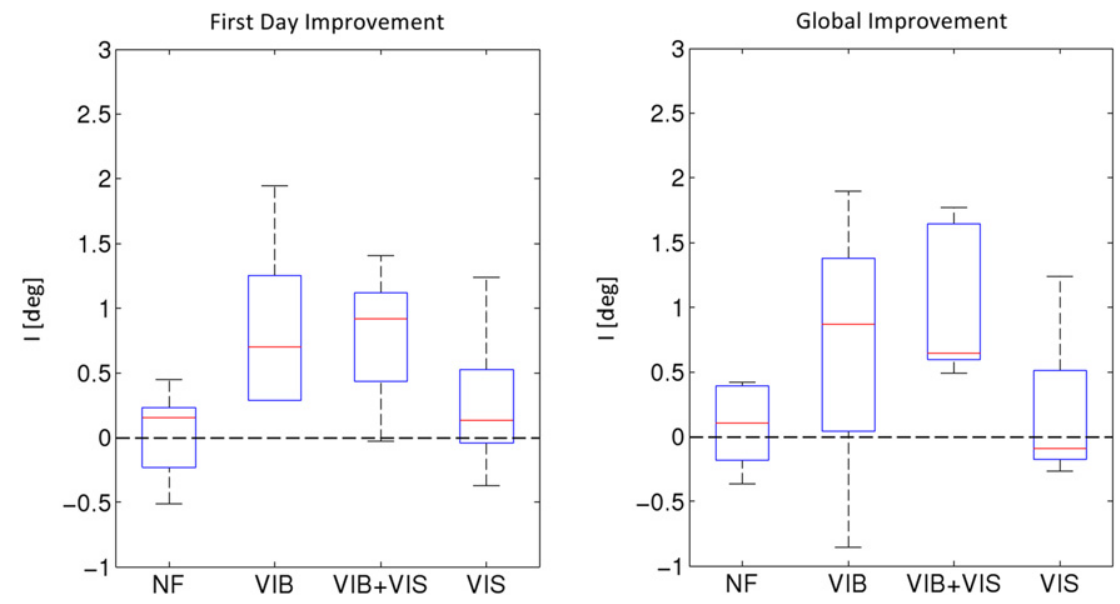

Fig. 5. Improvements of the four groups' participants between post day $1 /$ day 2 and initial test.

Training of body limbs motion does need full kinematics features, therefore it can be carried out both on the full and the lightweight platforms. However back and arms motion are required, therefore a motion tracking system has to be used. Timing training does not require a precise model of the boat, that is implemented mainly for visualization purposes, whereas it is necessary to accurately model the rower to have a precise yet fast estimation of her/his motion.

Expert rowers performances on the SPRINT platform were analyzed in order to find quantitative indices of the temporal structure which could be used for timing training. Performance analysis carried out to have a digital representation of the temporal pattern of the drive phase is shown in [9] and it is not reported here. From the analysis it emerged that two parameters describe the experts' timing: $t_{b}$ for back swing onset and $t_{a}$ for arms bending onset. These parameters are defined as

$t_{b}=\frac{T_{b}}{T_{d}-T_{0}} \quad$ and $\quad t_{a}=\frac{T_{a}}{T_{d}-T_{0}}$

where $T_{b}$ is the time of back swing onset, $T_{a}$ is the time of arms bending onset, $T_{0}$ is the time of legs pull onset and $T_{d}$ is the drive phase end time. Fig. 6 shows the correct time structure according to Adams style [16] along with previously mentioned parameters. Block performance regarding back swing and arms bending was separately scored. Scores increased as the ratios of correct strokes over the total. Timing errors are defined as

$e_{b}=t_{b}-\tilde{t}_{b}$ and $e_{a}=t_{a}-\tilde{t}_{a}$

for back and arms respectively, where $\tilde{t}_{b}$ and $\tilde{t}_{a}$ are the target values of timing parameters. Since these parameters are mostly based on angles and limb lengths normalized with respect to their maximum value, it is enough to put the trainee in a calibration position at the beginning of the training session to compensate for the dependence of the performance indices on the user's size. The calibration pose as well as the performance indices were tested by means of pivot sessions with differently sized participants to assess their effectiveness in scoring performance. Since the time the action is performed is the variable taken into account for training, it was checked that the information processing path (VICON capturing-PC processing-feedback) was fast enough to provide reliable information for training.

Trainees are provided with visual cues, audio cues and vibrotactile feedback. The visual cue is the SPRINT Virtual Environment where the stroke pace is shown. The audio cue is made of two beeps played by speakers at $T_{b}$ and $T_{a}$, the times the

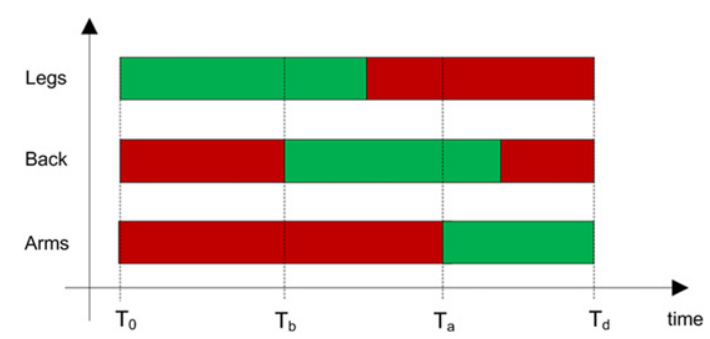

Fig. 6. Time structure of the rowing drive phase. The green zones are the time lapses in which the limb is actively moved to propel the boat. Conversely, the limb should not be moved in the red time lapses. (For interpretation of the references to colour in this figure legend, the reader is referred to the web version of this article.)

back and arms should be moved to have perfect timing. Vibrotactile feedback is given by vibrating motors worn on the wrist and the back. Vibration is triggered when the timing error exceeded the initially set thresholds, namely $\tilde{e}_{b}$ and $\tilde{e}_{a}$. Online feedback is provided immediately after the error is performed. According to the expertise of the participants, some task simplifications may be introduced: thresholds for error triggering can be set higher than experts' performance and variability and load can be completely removed. Since correct timing requires a considerable trainee's effort and the task is evaluated only once per stroke, blocks' length is set high (at least two minutes) to have several strokes even at low pace but, at the beginning, load is set to zero (R0 in Fig. 8) to ease novices performing the required acceleration without getting fatigued. Rest time between workout blocks may be used to show trainees the ratio of correct back/arms trials as performance indices.

The experiment carried out to evaluate the timing accelerator is here summarized. A more detailed description of the analysis and evaluation activities can be found in [17].

Eight naive adults (aged $26.1 \pm 4.9$ ) participated in the experiment. The task consisted of rowing on the SPRINT platform following the timing given by the auditory cue at $T_{b}$ and $T_{a}$. They were asked to keep their pace in the interval 15-18 strokes per minute (spm). Four participants were assigned to the vibrotactile provided group (VIB-KR) whereas the others to the knowledge of results only group (KR). The former were given the vibrational feedback during training blocks, the latter were not. All participants received knowledge of results after each training block. The experiment lasted three days. Pre-post training assessment blocks were performed to assess training effectiveness. Details about the final protocol, duration, rest time and load 


\begin{tabular}{ccccccc}
\hline Day & Session & Type & Load & Blocks & Duration & $\begin{array}{c}\text { Rest } \\
\text { Time }\end{array}$ \\
\hline \multirow{4}{*}{1} & S1 & Pre & No & 4 & 1, & $1, / 2$ \\
& S2 & Tra & No & 10 & 2, & $1, / 2$, \\
& S3 & Pos & No & 4 & 1, & $1, / 10^{\prime}$ \\
& S4 & Pos & Full & 4 & 1, & 1, \\
\hline \multirow{4}{*}{2} & S5 & Pre & No & 4 & 1, & $1, / 10^{\prime}$ \\
& S6 & Pre & Full & 4 & 1, & $1, / 2$, \\
& S7 & Tra & Full & 10 & 2, & $1, / 2$ \\
& S8 & Pos & Full & 4 & 1, & $1, / 10^{\prime}$ \\
& S9 & Pos & No & 4 & 1, & 1, \\
\hline 3 & S10 & Ret & Full & 4 & 1, & 1 \\
\hline
\end{tabular}

Fig. 7. Timing training protocol. Pre, Pos and Tra refer respectively to preliminary assessment sessions, post training assessment session and training sessions. The last rest time of each rest time cell has been adopted for the last workout block of the session.

condition are shown in Fig. 7. Errors $e_{a}(i)$ and $e_{b}(i)$ were used for the evaluation: total error

$\Delta G(i)=\sqrt{e_{a}^{2}(i)+e_{b}^{2}(i)}$

is a performance index that takes into account both back and arms timing errors. Fig. 8 shows the average of total error $\Delta G(i)$ for each block of each test session for all participants. Both $\mathrm{VIB}+\mathrm{KR}$ and KR group participants generally improve. It is possible to note that arms error is generally lower than backs one and that lower errors are produced in no load conditions. From the graphs vibrotactile feedback does not seem to give further benefits when coupled with audio guidance and KR. Data were further analyzed in order to check the effectiveness of vibrotactile feedback, effects of resistance, focus on the goal and to find common behaviors among subjects. The details of the analysis are presented in [17]. The main results are reported in Fig. 8. This experiment showed that if audio guidance, vibrotactile feedback and knowledge of results are concurrently provided, the effects of vibrotactile feedback are not noticeable. However, from this experiment some interesting considerations useful for the timing training design were drawn. First of all KR should be removed in order to amplify the effect of vibrotactile feedback. Then, since load condition strongly interferes with novice participants training, the next participants will experience only one low level of load. Finally, we saw that arms are generally better controlled than back (lower errors), therefore an improvement of learning is likely to happen if, at the beginning of training, instructions focus the trainees attention on the back.

\section{Conclusions}

The organization of a platform for sport training involves the combination of several competences, integrating motor science, mechanical engineering, biomechanics and computing for providing an overall experience in which different training scenarios are possible and in which users of different levels can be trained. Such organization is centered around multiple levels of training loops from the low level interaction to the high level long term training protocol. What emerges from the presentation of the rowing system is indeed the variety of the approaches undertaken to evaluate technique training. Common to these different approaches is a model of the feedback in the VE for supporting training and the identification of performance indices based on models taken from experts using heuristics of machine learning techniques.

There are two challenges not yet covered in this discussion. The first deals with the reuse of results, algorithms and feedback across tasks and skills, and the second is the measure of the transfer of training from the simulated environment to the real environment. This latter is currently in progress for the rowing system aiming at estimating the transfer capability of the system.

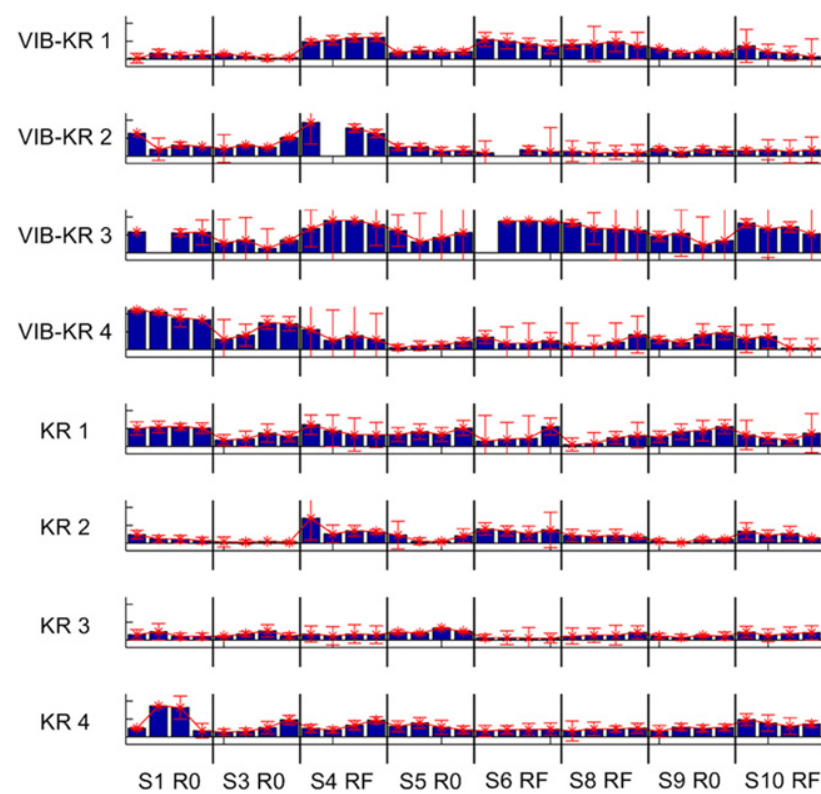

Fig. 8. $\Delta G(i)$ of all the subjects during test sessions.

\section{References}

[1] H. Miles, S. Pop, S. Watt, G. Lawrence, N. John, A review of virtual environments for training in ball sports, Computers \& Graphics 36 (6) (2012) 714-726.

[2] B. Bideau, R. Kulpa, N. Vignais, S. Brault, F. Multon, C. Craig, Using virtual reality to analyze sports performance, IEEE Computer Graphics and Applications 30 (2) (2010) 14-21.

[3] E. Ruffaldi, A. Filippeschi, A. Frisoli, C.A. Avizzano, B. Bardy, D. Gopher, M. Bergamasco, Sprint: a training system for skills transfer in rowing, in: T. Gutiérrez, E. Sánchez (Eds.), SKILLS09 International Conference on Multimodal Interfaces for Skills Transfer, CEIT- Centro de Estudios e Investigaciones Técnicas de Gipuzkoa, Bilbao, Spain, 2009, ISBN:978-84-6135456-5.

[4] A. Shepherd, Hierarchical task analysis and training decisions, Programmed Learning and Educational Technology 22 (2) (1985) 162-176.

[5] N. Dahlstrom, S. Dekker, R. Van Winsen, J. Nyce, Fidelity and validity of simulator training, Theoretical Issues in Ergonomics Science 10 (4) (2009) 305-314.

[6] E. Ruffaldi, A. Filippeschi, C. Avizzano, B. Bardy, D. Gopher, M. Bergamasco, Feedback, affordances and accelerators for training sports in virtual environments, MIT Presence 20 (1) (2011).

[7] C. Cruz-Neira, D.J. Sandin, T.A. DeFanti, Surround-screen projection-based virtual reality: the design and implementation of the CAVE, in: SIGGRAPH'93 Proceedings of the 20th Annual Conference on Computer Graphics and Interactive Techniques, 1993, pp. 135-142. http://dx.doi.org/10.1145/166117.166134.

[8] A. Williams, et al., Skill Acquisition in Sport, Routledge, 2005.

[9] Alessandro Filippeschi, Emanuele Ruffaldi, Expert rowers' motion analysis for synthesis and technique digitalization, BIO Web of Conferences 1 (2011) 00024. http://dx.doi.org/10.1051/bioconf/20110100024.

[10] Leonard Johard, Alessandro Filippeschi, Emanuele Ruffaldi, Real-time error detection for a rowing training system, BIO Web of Conferences 1 (2011) 00044. http://dx.doi.org/10.1051/bioconf/20110100044.

[11] C.P. Hoffmann, A. Filippeschi, E. Ruffaldi, S. Blanc, L. Verbrugge, B.G. Bardy, Mastering energy management during rowing using virtual reality, BIO Web of Conferences 1 (2011) 35. http://dx.doi.org/10.1051/bioconf/20110100035.

[12] F. Bissmark, H. Nakahara, K. Doya, O. Hikosaka, Responding to modalities with different latencies, Advances in Neural Information Processing Systems 17 (2005) 169-176

[13] G. Wulf, C.H. Shea, Principles derived from the study of simple skills do not generalize to complex skill learning, Psychonomic Bulletin \& Review 9 (2) (2002) 185-211.

[14] E. Ruffaldi, A. Filippeschi, O. Gonzales, D. Gopher, Visuo-vibrotactile trajectory training in rowing experiment, in: SKILLS 09 Enaction on SKILLS, 2009.

[15] V. Kleshnev, Boat acceleration, temporal structure of the stroke cycle, and effectiveness in rowing, Journal of Sports Engineering and Technology 224 (1) (2010) 63-78.

[16] V. Nolte, et al., Rowing Faster, Human Kinetics Publishers, 2005.

[17] A. Filippeschi, E. Ruffaldi, M. Korman, Preliminary evaluation of timing training accelerator for the SPRINT rowing system, BIO Web of Conferences 1(2011) 25. http://dx.doi.org/10.1051/bioconf/20110100025. 


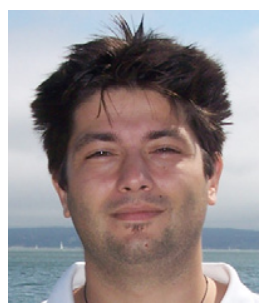

Emanuele Ruffaldi (Eng., Ph.D.) is Assistant Professor in Applied Mechanics of the PERCRO Lab, Scuola Superiore S. Anna, Pisa, Italy. He obtained his Ph.D. on perceptual robotics in 2006 from Scuola Superiore S. Anna discussing a thesis on perceptually inspired Haptic Algorithms. His research interests are in the application of machine learning for modeling behaviour of humans with a focus on skill training in Virtual Environments and integration with robot learning. He is involved in the domain of haptics with a focus on haptic rendering. He participated in the research activities of the European project SKILLS IP as workpackage leader for the Rowing Demonstrator (www.skills-ip.eu/row). Inside SKILLS he has focused research on two aspects: on the one hand the issues of combining the modeling of the skill with the creation of appropriate training feedback, the other on the digital representation of human skills combined with issues of data management. He has contributed to previous EU projects like ENACTIVE NoE, Decision In Motion, HAPTEX, CREATE, and PureForm. Emanuele has been the author of more than 60 papers published in proceedings of scientific conferences and journals. He has been the general chair of the ENACTIVE08 and the Program Chair of IEEE RO-MAN 2010.

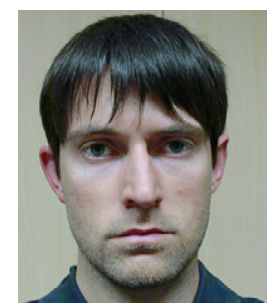

Alessandro Filippeschi (Eng.), is a Ph.D. student in perceptual robotics at the PERCRO Lab, Scuola Superiore S. Anna, Pisa, Italy. He received a M.Sc. in mechanical engineering in 2007, discussing a thesis on the mechanical design of a rowing simulator. Dr. Filippeschi's current research focuses on analysis, design and implementation of systems for the human motion skills enhancement and/or rehabilitation, it is part of the research activities of the European project SKILLS IP. He is the author of 4 papers published in peer-reviewed journals or proceedings. 